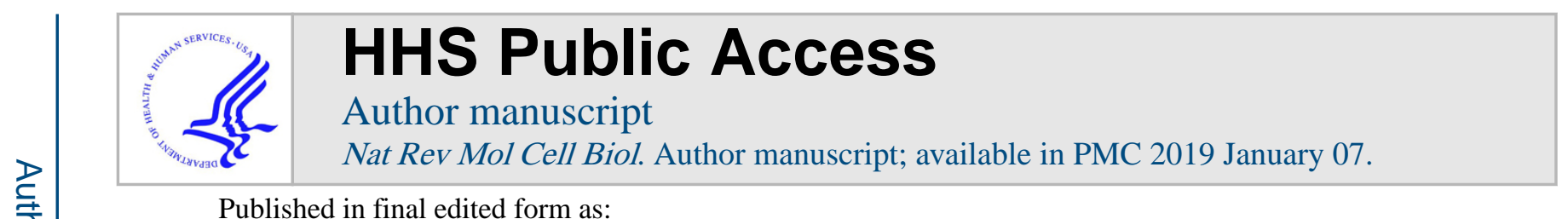

Published in final edited form as:

Nat Rev Mol Cell Biol. 2014 February ; 15(2): 79. doi:10.1038/nrm3740.

\title{
THE LIPID TRADE
}

\section{William A. Prinz}

National Institutes of Health and National Institute of Diabetes and Digestive and Kidney Diseases, Laboratory of Cell and Molecular Biology, 8 Center Drive, Bethesda, Maryland 20892, USA

Lipid transport proteins (LTPs) were first purified more than 25 years ago on the basis of their remarkable ability to facilitate lipid exchange between membranes in vitro. There are at least five superfamilies of LTPs and all cells express multiple LTPs. These proteins have crucial roles in vesicular trafficking, signalling and lipid metabolism, especially in phosphoinositide metabolism.

But what do LTPs actually do in cells? Addressing this question has been surprisingly challenging. They contain a hydrophobic pocket that enables them to bind single lipids, indicating that they might shuttle lipids between membranes. Some LTPs have been suggested to function as lipid sensors rather than transporters.

A groundbreaking study from the Bankaitis group (Schaaf et al., 2008) provided evidence for a third possible function for LTPs and, more importantly, revealed a pivotal new aspect of how most LTPs operate. The authors focused on Sfh1, a member of the large Sec14-like family of LTPs that can bind either phosphatidylinositol (PtdIns) or phosphatidylcholine (PC).

They argued that, rather than sensing or transporting PtdIns, Sfh1 presents it to PtdIns kinases, stimulating phosphoinositide production. They also showed that binding of Sfh1 to $\mathrm{PC}$ and PtdIns is mutually exclusive and, remarkably, that Sfh1 must have the capacity to bind to both lipids to be functional in cells, which suggests that LTPs exchange one lipid for another. This property allows them to function in ways that are more sophisticated than previously imagined. For example, as shown in three later studies (de Saint-Jean et al., 2011; Kono et al., 2013; Mesmin et al., 2013), some LTPs exchange a phosphoinositide for a second lipid, which allows them to exploit differences in the phosphoinositide concentration between two membranes to drive the directional transport of the second lipid. In the case of Sfh1 and other similar LTPs, PC exchange for PtdIns might facilitate PtdIns presentation to kinases, and this might depend on the relative abundance of PtdIns and PC in a membrane.

Schaaf et al. was the first study to demonstrate that some LTPs exchange one lipid for a second lipid, indicating that LTPs are more than simple transporters.

wp53m@nih.gov.

The author declares no competing interests. 


\section{ORIGINAL RESEARCH PAPERS}

Schaaf $\mathrm{G}$ et al. Functional anatomy of phospholipid binding and regulation of phosphoinositide homeostasis by proteins of the sec14 superfamily. Mol. Cell 29, 191-206 (2008) [PubMed: 18243114]

de Saint-Jean MV et al. Osh4p exchanges sterols for phosphatidylinositol 4-phosphate between lipid bilayers. J. Cell Biol 195, 965-978 (2011) [PubMed: 22162133]

Kono $\mathrm{N}$ et al. Impaired a-TTP-PIPs interaction underlies familial vitamin E deficiency. Science 340, 1106-1110 (2013) [PubMed: 23599266]

Mesmin B et al. A four-step cycle driven by PI(4)P hydrolysis directs sterol/PI(4)P exchange by the ER-Golgi tether OSBP. Cell 155, 830-843 (2013) [PubMed: 24209621] 